\title{
Unintended Consequences of Sensor, Signal, and Imaging Informatics: New Problems and New Solutions
}

\author{
C. Hughes', S. Voros 2,4, A. Moreau-Gaudry, \\ 1 Univ. Grenoble Alpes, INSERM, CIC 1406; CHU Grenoble, Pôle Recherche, Grenoble, France \\ 2 Univ. Grenoble Alpes, CNRS, INSERM, TIMC-IMAG, Grenoble, France \\ 3 INSERM CIC 1406; Univ. Grenoble Alpes, CNRS, TIMC-IMAG; CHU de Grenoble, Pôle Santé \\ Publique, Grenoble, France \\ 4 Section Editors for the IMIA Yearbook Section on Sensor Signal and Imaging Informatics
}

\section{Introduction}

Although innovative approaches are increasingly being developed to improve the care of patients, unintended consequences are emerging with new medical challenges. This synopsis aims at reporting the main articles that have been identified in the field of sensor, signal and imaging informatics to tackle these challenges and the new emerging solutions that have been proposed. The selection was performed using PubMed and Web of Science databases, according to a generic MesH keyword query provided by experts.

\section{Results}

\section{Keywords}

Innovative technology, unintended consequences, novel solutions

Yearb Med Inform 2016:159-62

http://dx.doi.org/10.15265/IY-2016-053

Published online November 10, 2016
Following the methodology described in the 2014 Yearbook, 531 articles were automatically selected, 30 were then pre-selected as candidate best papers in a double-blind review process. From these, 15 were selected, with the four best papers selected by external review. With advances in technology, the selection shows a general tendency to acquire a greater quantity of ever more accurate data with the emergence of new challenges. From a diagnosis point of view, these challenges are in relation to image improvement and more accurate classification. From a therapeutic point of view, minimally-invasive therapeutic procedures require the development of "augmented solutions" in order to further improve safety.

\section{Discussion}

\section{Imaging Improvement for Better Patient Care}

Imaging is an ideal tool for diagnosis and decision making. Nevertheless, the increased use of computed tomography (CT), raises concern about radiation-associated cancer risks, shifting the focus from technical innovation to the minimization of radiation exposure by more effective dose control. However, low dose CT images suffer from severe artifacts and reduced feature details. The problem is to reduce the radiation exposure while maintaining standard image quality. [1] proposes a method of iterative reconstruction in combination with an integrated circuit detector. Tested on head CT images, acquired with nearly $50 \%$ dose reduction, the contrast-tonoise ratio and the image sharpness were fully preserved in the low-dose images. [2] shows that a high quality restoration of low-dose CT images can be achieved using a database of diverse prior full-dose CT images in combination with a localized similarity scheme. The method was tested using CT images of the lung, with lowdose images generated by the addition of Gaussian noise.

Many different imaging modalities are now necessary to optimize the diagnosis step. The human mental process to precisely combine the multi-modal data has now become too complex. In particular, 2D-3D (i.e. slice to volume) registration requires the development of specific meth- 
ods, [3] proposes a non-rigid method that associates two distinct graphs to the plane selection and the in-plane deformations. Tested on a multimodal brain dataset (2D ultrasound (US) and 3D CT images), the method achieved state-of-the-art results, while decreasing the computation time. Another example concerns hybrid imagers that combine positron emission tomography (PET) with magnetic resonance (MR) imaging to improve the diagnosis that is possible using just one of the modalities. However, the artefacts created by the required PET attenuation correction may negatively impact the PET interpretation. The study carried out by [4] showed that though attenuation map artifacts were found in a considerable percentage of PET/MR datasets, according to the data studied, these artefacts did not alter the diagnosis made. However, attenuation maps should be carefully checked for artefacts, and when present, the native PET images should be taken into account.

Another unintended consequence of greater access and increased use of imaging data, is a demand for US image quality improvement. The problem is that properties such as speckle noise mask meaningful texture, therefore degrading the contrast resolution, meaning that the clarity of edges and borders is reduced, with potential to interfere with diagnosis. [5] proposes a solution which includes an adaptive bas-relief filter to suppress speckle combined with an edge map to enhance edges and weak borders.

\section{New Solutions for Early Stage Diagnosis and Disease Classification}

Alongside these increasingly sophisticated imaging techniques, there is a need to improve decision-making, and ideally at an early stage of the disease to prevent potentially morbid complications. For instance, foot diseases are a common complication of diabetes, for which an early detection can prevent devastating consequences (amputation, mortality). An automated foot assessment solution is thus investigated by [6] in which a color and thermal image of both feet is acquired. The foot region is detected in the color image, which is then registered to the thermal image. The two feet are then

Table 1 Best paper selection of articles for the IMIA Yearbook of Medical Informatics 2016 in the section 'Sensor, Signal and Imaging Informatics'. The articles are listed in alphabetical order of the first author's surname.

\section{Section}

Sensor, Signal and Imaging Informatics

- Chen Z, Strange H, Oliver A, Denton ER, Boggis C, Zwiggelaar R. Topological Modeling and Classification of Mammographic Microcalcification Clusters. IEEE Trans Biomed Eng 2015;62(4):1203-14.

- Ha S, Mueller K. Low dose CT image restoration using a database of image patches. Phys Med Biol 2015;60(2):869-82.

- Liu C, van Netten JJ, van Baal JG, Bus SA, van der Heijden F. Automatic detection of diabetic foot complications with infrared thermography by asymmetric analysis. J Biomed 0pt 2015;20(2):26003.

- Mert S, Özbek E, Ötünçtemur A, Çulha M. Kidney tumor staging using surface-enhanced Raman scattering. J Biomed Opt 2015;20(4):47002

registered and the temperature difference is calculated, zones with a $2.2^{\circ} \mathrm{C}$ temperature difference are considered at risk.

In the field of breast imaging, the analysis of microcalcification clusters can enable an early diagnosis of breast cancer. However, radiologists encounter difficulties in classifying such clusters as malignant or benign. A solution that enables the automatic classification according to topology and connectivity at different scales is proposed by [7], with good results obtained when tested on three different datasets. In the same way, in the field of kidney cancer, there is a need to precociously differentiate malignant from benign tissue, with stage determination. [8] demonstrates that surface-enhanced Raman scattering (SERS) in conjunction with a principal component analysis (PCA) and linear discriminant analysis (LDA) classification algorithm, has the ability to differentiate biopsies as benign or malignant and furthermore, in the latter case, to identify the stage of the tumor, by exploiting the differences in the biochemical components reflected in the SERS spectra.

Finally, a unified framework for joint clustering and component analysis of point sets proposed by [9] is capable of clustering MR cardiac datasets into normal healthy subjects, patients with pulmonary hypertension and patients with hypertrophic cardiomyopathy, as well as extracting the intra-cluster variations. The method is capable of handling point sets with no point-to-point correspondences and automatically identifying the number of clusters, outperforming traditional PCA based statistical shape models in generalization and specificity.

\section{Augmented Environment for Safer Minimally-invasive Medico-surgical Processes}

Minimally invasive approaches suffer from a lack of contextual (e.g. anatomical, tissue quality) information that is necessary to improve their safety. In the case of a brain needle biopsy, the rupture of a blood vessel during excision is a significant risk, where hemorrhaging could cause neurological deficit or death. There is thus a need to provide in-procedure guidance that alerts surgeons to the presence of significant blood vessels in the vicinity of the needle. The solution proposed by [10] demonstrates a proof-ofconcept that interstitial optical tomography is capable of detecting blood vessels $\sim 1.5$ $\mathrm{mm}$ around the needle.

For the case of US guided radiofrequency (RF) ablation, improved needle tracking has been achieved by embedding electromagnetic position sensors (EMPS) at the tip of the RF electrode. [11] demonstrates that the EMPS RF electrode allows for faster electrode placement into target lesions than a conventional electrode, while maintaining comparable ablation performance. For surgical process involving ultrashort-pulsed lasers, [12] has developed and tested a real-time feedback system by which the material being ablated by the laser is detected and identified from laser-induced breakdown spectroscopy signals, enabling the laser parameters to be adjusted accordingly.

Another challenge is to be able to accurately localize minimally-invasive technology within the body. For instance, wireless capsule endoscopy (CE) is a minimally invasive 
technique that has been established as the prime-imaging tool in the investigation of small-bowel diseases. The conventional capsule has been equipped with three miniature legs ending in wheels; the wheel rotations are registered, enabling the travelled distance to be computed in order to accurately localize the $\mathrm{CE}$ along the digestive tract [13].

\section{Innovative Technology to Compensate Vision and Communication Impairment}

A further aspect of research is the potentiality of "augmenting" the capabilities of the patient by using minimally-invasive technology to compensate for a certain impairment. Recent work has been carried out to investigate the restoration of vision by means of a retinal prosthesis for patients with certain types of vision impairment. However, a significant limitation is the sensor resolution, with the challenge being the miniaturization process. By using a three transistor complementary metal-oxide-semiconductor (CMOS) image sensor and by simplifying the stimulation-current generating circuit, [14] has shown that a $43.9 \%$ pixel size reduction can be achieved, compared to the current state-ofthe-art. Such a size reduction would enable more than 5000 pixels to be implemented in the available area allowing a sufficient spatial resolution to read sentences and to recognize a face.

To enable communication by individuals with severe neuromuscular disabilities, brain signals in electroencephalography (EEG) data can be used by a brain-computer interface (BCI). However, spelling is slow, as the data is averaged over multiple trials to increase the signal-to-noise (SNR) ratio. [15] developed an algorithm to increase the communication rate, while maintaining accuracy, by adaptively selecting the number of trials based on the acute SNR of a user's EEG data, with further improvement achieved by incorporating information about the user's language. The algorithm was validated in target BCI users, with significant performance improvements measured compared with current state-of-the-art methods.

\section{Conclusion \& Outlook}

The impressive progress made in the field of medical technology enables new and more relevant therapeutic approaches to be developed. These innovative approaches should justifiably be promoted but also accurately evaluated to rapidly detect any possible problems, that could emerge as they are adopted by the medical and patient community. Our final aim must remain the improvement of patient care using innovative technology but in compliance with ethics and health-economic policies.

\section{References}

1. Brodoefel H, Bender B, Schabel C, Fenchel M, Ernemann U, Korn A. Potential of combining iterative reconstruction with noise efficient detector design: aggressive dose reduction in head CT. $\mathrm{Br}$ J Radiol 2015;88(1050):20140404.

2. Ha S, Mueller K. Low dose CT image restoration using a database of image patches. Phys Med Biol 2015;60(2):869-82.

3. Ferrante E, Fecamp V, Paragios N. Slice-to-volume deformable registration: efficient one-shot consensus between plane selection and in-plane deformation. Int J Comput Assist Radiol Surg 2015;10(6):791-800.

4. Brendle C, Schmidt H, Oergel A, Bezrukov I, Mueller M, Schraml C, et al. Segmentation-based attenuation correction in positron emission tomography/magnetic resonance: erroneous tissue identification and its impact on positron emission tomography interpretation. Invest Radiol 2015;50(5):339-46.

5. Du H, Ma R, Wang X, Zhang J, Fang J. Bas-Relief Map Using Texture Analysis with Application to Live Enhancement of Ultrasound Images. Ultrasound Med Biol 2015;41(5):1446-60.

6. Liu C, van Netten JJ, van Baal JG, Bus SA, van der Heijden F. Automatic detection of diabetic foot complications with infrared thermography by asymmetric analysis. J Biomed Opt 2015;20(2):26003.

7. Chen Z, Strange H, Oliver A, Denton ER, Boggis C, Zwiggelaar R. Topological Modeling and Classification of Mammographic Microcalcification Clusters. IEEE Trans Biomed Eng 2015;62(4):1203-14.

8. Mert S, Özbek E, Ötünçtemur A, Çulha M. Kidney tumor staging using surface-enhanced Raman scattering. J Biomed Opt 2015;20(4):47002.

9. Gooya A, Lekadir K, Alba X, Swift AJ, Wild JM, Frangi AF. Joint clustering and component analysis of correspondenceless point sets: application to cardiac statistical modeling. In: Information Processing in Medical Imaging. Springer; 2015. p. 98-109.

10. Goyette A, Pichette J, Tremblay M-A, Laurence A,
Jermyn M, Mok K, et al. Sub-diffuse interstitial optical tomography to improve the safety of brain needle biopsies: a proof-of-concept study. Opt Lett 2015;40(2):170-3.

11. Kang TW, Lee MW, Choi SH, Rhim H, Lim S, Song KD, et al. A novel electrode with electromagnetic tip tracking in ultrasonography-guided radiofrequency ablation: A phantom, ex vivo, and in vivo experimental study. Invest Radiol 2015;50(2):81-7.

12. Huang H, Yang L-M, Bai S, Liu J. Smart surgical tool. J Biomed Opt 2015;20(2):28001.

13. Karargyris A, Koulaouzidis A. OdoCapsule: next-generation wireless capsule endoscopy with accurate lesion localization and video stabilization capabilities. IEEE Trans Biomed Eng 2015;62(1):352-60.

14. Oh S, Ahn J-H, Lee S, Ko H, Seo JM, Goo Y-S, et al. Light-Controlled Biphasic Current Stimulator IC Using CMOS Image Sensors for High-Resolution Retinal Prosthesis and In Vitro Experimental Results With rd1 Mouse. IEEE Trans Biomed Eng 2015;62(1):70-9.

15. Mainsah BO, Collins LM, Colwell KA, Sellers EW, Ryan DB, Caves K, et al. Increasing BCI communication rates with dynamic stopping towards more practical use: an ALS study. J Neural Eng 2015;12(1):016013.

\section{Correspondence to:}

Sandrine Voros

E-mail:sandrine.voros@imag.fr

Alexandre Moreau-Gaudry

E-mail: alexandre.moreau-gaudry@imag.fr

\section{Appendix: Content Summa- ries of the Selected Best Pa- pers for the IMIA Yearbook 2016, Section Sensor, Signal and Imaging Informatics}

\section{Ha S, Mueller K \\ Low dose $C T$ image restoration using a database of image patches}

\section{Phys Med Biol 2015;60(2):869-82}

An important current issue in the field of $\mathrm{CT}$ is to reduce radiation exposure by more effective dose control while maintaining standard image quality. Low-dose CT images suffer from severe noise artifacts and reduced feature details, impeding diagnosis capability. In order to achieve lose-dose CT restoration, the authors propose the development of a database of diverse, high-quality CT image 
patches, obtained from prior regular-dose scans. For each noisy pixel, a set of suitable patches is retrieved from the database by similarity matching, and are then applied using a Non-Local Means type similarity-weighted filter. The use of image patches is attractive as the non-trivial problem of image registration is avoided and storing patches rather than images means that redundancy can be removed, enabling greater diversity to be stored. Furthermore, it is shown that the patches can be stored without anatomical tags, as the statistics of the patches are sufficiently strong to yield high quality matches, producing high quality image restorations. The proposed method performs significantly better than current denoising approaches.

\section{Liu C, van Netten JJ, van Baal JG, Bus SA, van der Heijden $F$}

\section{Automatic detection of diabetic foot complications with infrared thermography by asymmetric analysis}

\section{J Biomed Opt 2015;20(2):26003}

Foot diseases are a common complication of diabetes and their early detection is essential in order to avoid devastating consequences. However, frequent risk assessment by health care professionals is impractical and manual examination by patients themselves is limited due to the various health impairments associated with diabetes. The authors present technology to carry out an automatic risk assessment of diabetic patients. Color and thermal images are acquired synchronously. The foot regions are segmented in the color image, a rigid transformation is calculated to align the color image with the thermal image and the left and right feet are registered using a non-rigid landmark based method. Zones where the temperature difference between the two feet is greater than $2.2^{\circ} \mathrm{C}$ are identified as being at risk. 76 diabetic patients with foot complications were included in the study, 35 out of 37 foot ulcers were successfully detected.

\section{Chen Z, Strange H, Oliver A, Denton ER, Boggis C, Zwiggelaar $R$ Topological Modeling and Classification of Mammographic Microcalcification Clusters \\ IEEE Trans Biomed Eng 2015;62(4):1203-14}

The presence of microcalcification clusters may be a primary sign of breast cancer. However, their classification is difficult and time consuming resulting in a high rate of unnecessary biopsies. The authors present a method for classifying microcalcification clusters in mammograms as malignant or benign based on the analysis of the topology and connectivity of individual microcalcifications within a cluster, using multiscale morphology. A set of graphs was generated to represent the topological structure at different scales. Eight graph metrics were extracted and their aggregation defined the multiscale topological feature vector used to classify the clusters using a k-nearest neighbors-based algorithm. The proposed method was evaluated using three datasets. High classification accuracy (up to $96 \%$ ) was achieved, outperforming current state-of-the-art methods.
Mert S, Özbek E, Ötünçtemur A, Çulha M Kidney tumor staging using surfaceenhanced Raman scattering

\section{J Biomed 0pt 2015;20(4):47002}

The early detection and the prediction of the tumor stage of kidney cancers is critical for diagnosis, choice of clinical treatment method and survival rate. The authors explore the possibility of using surface-enhanced Raman scattering (SERS) - in which a nano-structured noble metal substrate is used to enhance Raman scattering - as a potential technique for the classification (benign/malign) and identification of different tumor stages. SERS improves sensitivity such that the detection of a single molecule is possible, the differences in the biochemical components of normal and cancer tissues are therefore reflected in the SERS spectra. An algorithm combining principal component analysis (PCA) with linear discriminant analysis (LDA) is used to identify the significant spectral characteristics of each tissue type, in order to differentiate the tissue types. The method was evaluated using 40 normal and 40 pathological - T1, T2 and T3 stage - biopsy tissue samples obtained from kidney cancer patients, combined with colloidal-silver nanoparticles. The results suggest that SERS spectra from tissue samples, combined with PCA-LDA diagnostic classification algorithms, can be used to reach a clinical decision for the stage determination of kidney cancers. 